\title{
Interchange Reconnection Alfvén Wave Generation
}

\author{
B.J. Lynch ${ }^{1} \cdot$ J.K. Edmondson ${ }^{2} \cdot$ Y. Li $^{1}$
}

(C) Springer $\bullet \bullet \bullet$

\begin{abstract}
Given recent observational results of interchange reconnection processes in the solar corona and the theoretical development of the S-Web model for the slow solar wind, we extend the analysis of the 3D MHD simulation of interchange reconnection by Edmondson et al. (Astrophys. J. 707, 1427, 2009). Specifically, we analyze the consequences of the dynamic streamer-belt jump that corresponds to flux opening by interchange reconnection. Information about the magnetic field restructuring by interchange reconnection is carried throughout the system by Alfvén waves propagating away from the reconnection region, distributing the shear and twist imparted by the driving flows, including shedding the injected stress-energy and accumulated magnetic helicity along newly open fieldlines. We quantify the properties of the reconnection-generated wave activity in the simulation. There is a localized high-frequency component associated with the current sheet/reconnection site and an extended low-frequency component associated with the large-scale torsional Alfvén wave generated from the interchange reconnection field restructuring. The characteristic wavelengths of the torsional Alfvén wave reflect the spatial size of the energized bipolar flux region. Lastly, we discuss avenues of future research by modeling these interchange reconnection-driven waves and investigating their observational signatures.
\end{abstract}

Keywords: Magnetohydrodynamics; Magnetic fields, Corona; Magnetic Reconnection, Theory; Solar Wind; Waves, Magnetohydrodynamic

\section{Introduction}

The interchange reconnection (IR) model for the solar wind was introduced by Fisk and co-authors to explain in-situ observations of the interplanetary magnetic field (Fisk, Zurbuchen, and Schwadron, 1999; Fisk and Schwadron, 2001; Fisk, 2005; Fisk and Zurbuchen, 2006) and various properties of the insitu slow solar wind, including its high variability, lateral extent, elemental

\footnotetext{
1 Space Sciences Laboratory, University of California, Berkeley, CA 94720, USA email: blynch@ssl.berkeley.edu; yanli@ssl.berkeley.edu

2 Atmospheric, Oceanic and Space Sciences Department, University of Michigan, Ann Arbor, MI 48109, USA email: jkedmond@umich.edu
} 
and ionic composition (e.g., Geiss, Gloeckler, and von Steiger, 1995; Gosling, 1997; Zurbuchen, 2007). Antiochos et al. (2011) and colleagues developed the Separatrix Web (S-Web) model to bridge the gap between the quasi-steady and the IR solar wind models, incorporating relevant aspects of dynamic IR behavior via self-consistent MHD modeling and rigorous topological analysis of the solar corona's temporal evolution (see Linker et al., 2011; Titov et al., 2011).

There has also been a long history of the development and refinement of waveheating and turbulent dissipation models for the generation of the solar wind (e.g. Hollweg, 1986; Matthaeus et al., 1999; Cranmer, van Ballegooijen, and Edgar, 2007; Cranmer and van Ballegooijen, 2010; Ofman, 2004, 2010; Verdini et al., 2010). These tend to work pretty well for the fast solar wind emanating from coronal holes, and appear to be making progress towards reproducing some of the elemental and ionic composition properties of the slow wind (Cranmer, van Ballegooijen, and Edgar, 2007; Laming, 2004, 2009; Bryans, Landi, and Savin, 2009). However, for the most part, the wave-heating models start with an initial wave power spectrum and concentrate on the transformation and evolution of this spectra, calculating the turbulent cascade to higher frequencies and ultimately the deposition of this energy into plasma heating and acceleration, typically through various wave-particle interactions or resonances with particular ions.

The purpose of this paper is to extend the analysis of the IR simulation presented by Edmondson et al. (2009) to characterize the wave activity that occurs as a consequence of the IR opening of large, closed field-lines. We examining the material and energy fluxes at the open-closed field boundary and show that IR transports the twist component of the previously closed flux into the open field in the form of a large-scale torsional Alfvén wave. While our simulation is highly idealized and the footpoint shearing motions used to energize the configuration are not meant to model observed photospheric flow patterns, we feel the large-scale vortical boundary flow that imparts twist to the entire flux system is a convenient representation of an aspect of the Antiochos (2013) helicity condensation theory.

The Antiochos (2013) helicity condensation model explains the accumulation of shear and/or twist at polarity inversion lines. They describe an inverse cascade transportation process where small-scale twist emerges and reconnects to larger and larger scales. This process is naturally limited by the topological boundaries of the flux system; the large scale, accumulated shear/twist condenses at both the polarity inversion line and the separatrix boundary between open and closed field. While the low-lying, highly sheared fields of filament channels are a common occurrence, we do not observe highly sheared fields at the boundaries between open and closed flux. Antiochos (2013) argued that the helicity that tries to accumulate at the coronal hole boundaries is, in fact, transferred onto open field-lines through IR processes occurring at the open-closed interface, and thus regularly escapes the corona. This is a reasonable conjecture since the interplanetary magnetic field and its fluctuations are known to transport magnetic helicity (e.g., Smith, 1999 and references therein).

The structure of the article is as follows. In Section 2, we present a brief overview of the MHD simulation and describe the topological evolution of interchange reconnection, illustrate the nonlinear, large-scale torsional Alfvén wave 
generation and quantify the opening of previously closed flux. In Section 3 we quantify the associated material and energy fluxes. In Section 4 we examine the spatial extent and radial propagation of the IR-generated torsional Alfvén wave fluctuations through our computational domain and examine the spatial structure and properties of the wave power spectra. In Section 5, we make suggestions for future observational tests, and discuss improvements for future numerical simulation work in this area.

\section{Overview of the MHD Simulation}

\subsection{Numerical Methods and Initial Conditions}

The Edmondson et al. (2009) simulation was run with the Adaptively Refined MHD Solver (ARMS) code, developed by C. Richard DeVore and collaborators. ARMS calculates solutions to the 3D nonlinear, time-dependent MHD equations that describe the evolution and transport of density, momentum, and energy throughout the system, and the evolution of the magnetic field and electric currents (see e.g., DeVore and Antiochos, 2008). The numerical scheme used is a finite-volume, multidimensional flux-corrected transport (FCT) algorithm (DeVore, 1991). The ARMS code is fully integrated with the adaptive-mesh toolkit PARAMESH (MacNeice et al., 2000), to handle dynamic, solution-adaptive grid refinement and support an efficient multiprocessor parallelization.

Figure 1(a) shows the initial magnetic-field configuration of our system. This is the well-known 3D embedded bipole / null-point potential field in spherical coordinates, the simplest nontrivial 3D structure that facilitates magnetic reconnection in response to system energization. The solar-surface contour map plots the radial component of the magnetic field. There are two flux systems associated with the two polarity-inversion lines separated by a hemispherical separatrix dome where the continuous intersection with the solar surface is indicated as the ring of magenta dots. The yellow field lines show the extent of the AR flux system while the green field lines trace the boundary of the streamer-belt flux system. Representative field lines of the open field regions are drawn in blue. The inner and outer spine field lines are shown in magenta as well as the boundary of the separatrix dome in the $\phi=0$ plane. There is a 3D null point at the intersection of the spine field lines with the separatrix done. Figure 1(b) plots the maximum magnitude of the applied shearing flows in the negative polarity spot of the AR (colored red in panel a). The flow field is constructed to follow the contours of $B_{r}$ on the $r=R_{\odot}$ boundary so the normal flux distribution remains constant throughout the simulation. The shearing flow magnitude is roughly $6 \%$ of the global average Alfvén speed and is smoothly ramped up from $0 \leq t \leq$ $2000 \mathrm{~s}$, uniform from $2000 \leq t \leq 20000 \mathrm{~s}$, and ramped back down from 20000 $\leq t \leq 22000 \mathrm{~s}$. We refer to Edmondson et al. (2009) for details of the initial magnetic-field model, properties of the initial solar atmosphere, the functional form of the shearing profile, and details of the simulation boundary conditions. 

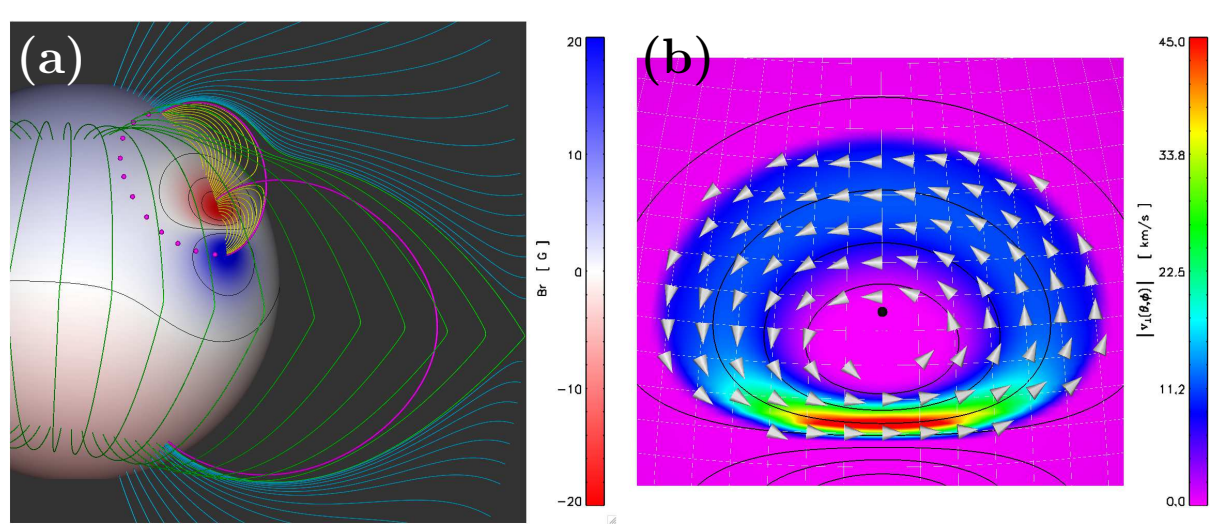

Figure 1. Panel (a) initial potential field showing the active-region flux (yellow field lines), the closed field streamer-belt boundary (green field lines) and the open field (blue field lines). The AR separatrix boundary on the solar surface is indicated with magenta dots, and the magenta fieldlines show the separatrix dome and inner and outer spine field lines. Panel (b) velocity profile shearing pattern applied to the red (negative) polarity AR flux. Adapted from Edmondson et al. (2009).

\subsection{Topological Evolution of Interchange Reconnection}

The magnetic topology and evolution of the Edmondson et al. (2009) simulation is essentially a large, spherical version of the twist-jet model (e.g., Shibata and Uchida, 1986; Pariat, Antiochos, and DeVore, 2009, 2010) and is qualitatively similar to the flux emergence scenario of Török et al. (2009). These authors have identified the outwardly propagating torsional Alfvén waves resulting from the reconnection dynamics and have suggested their potential contribution to coronal heating and the acceleration of the solar wind. The placement of the parasitic polarity spot near a well-defined open-closed field boundary (i.e., the edge of the helmet streamer belt) allowed Edmondson et al. (2009) to track the evolution of the open flux and the open-closed boundary during the interchange reconnection process and allows us to perform a quantitative analysis of the physical properties of the simulation outflow originating at the edge of the streamer belt.

The top row of Figure 2 illustrates both the interchange reconnection process occurring at the stressed null point and the propagation of the kinks and twist in the newly reconnected open field lines (the white lines). The planar surface shows the magnitude of current density, strongest in the core of the sheared active-region flux, but also outlining the current sheet that has formed along the separatrix boundary of the AR flux system. In each panel the white field lines are plotted from the same (stationary) footpoints on the solar surface so their evolution depicts the flux transfer of originally closed, stressed AR field through the current sheet and into the open field region where the stress and helicity (twist component) propagate towards the outer-radial boundary. The bottom row of Figure 2 shows the spatial distribution of the open flux footpoints on the lower $r=R_{\odot}$ boundary at each corresponding time. The narrow, open field corridor is in the process of opening up along the entire separatrix boundary as the outer spine line becomes open (as discussed in Edmondson et al., 2009). 

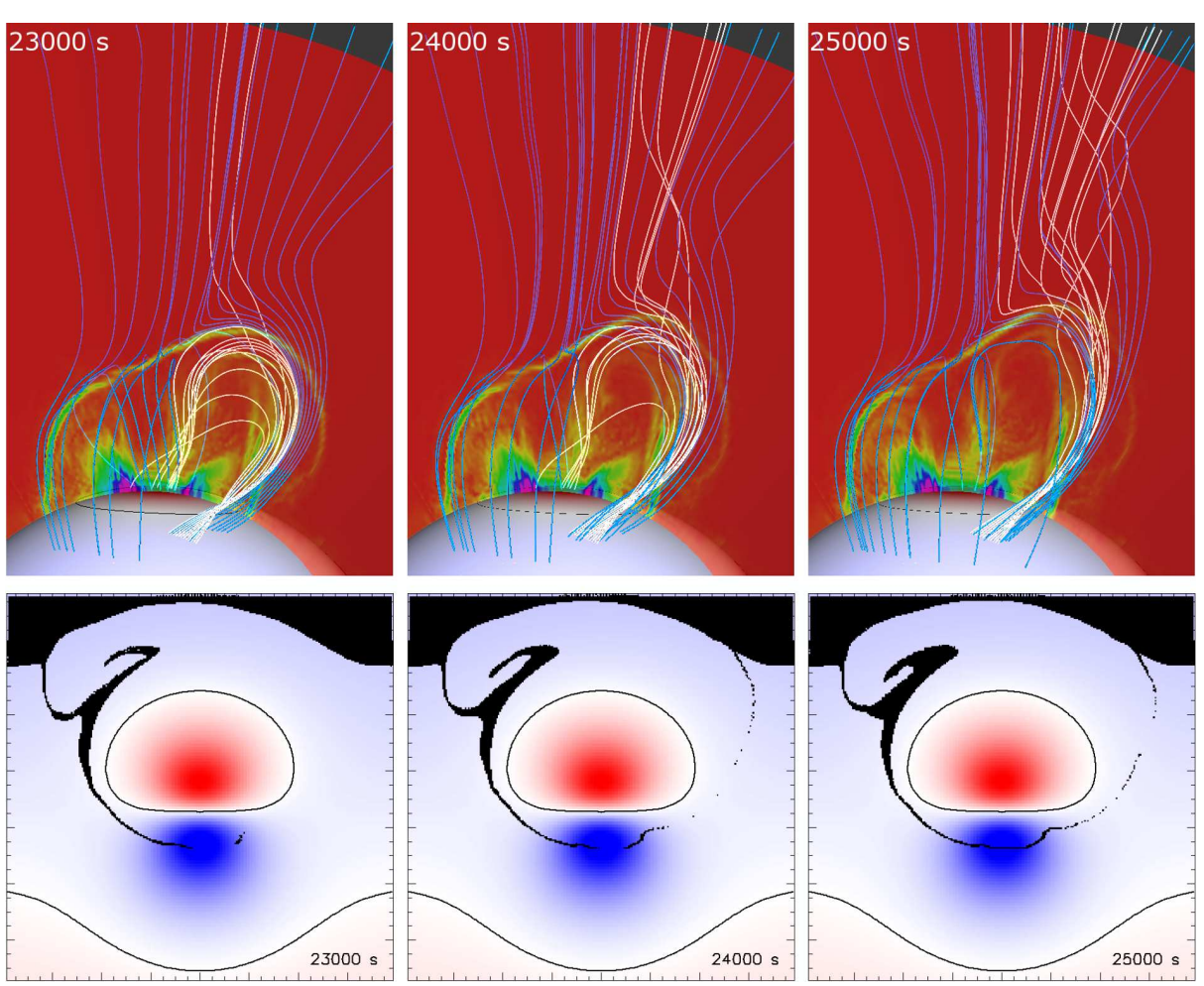

Figure 2. Top row, Heliospace renderings of the simulation data. The white field lines traced from $r=R_{\odot}$ footpoints are the same field lines in each panel against backdrop of current density magnitude. Bottom row, frames of $B_{r}\left(R_{\odot}, \theta, \phi\right)$ with the open flux area area overplotted as the black pixel map showing the evolution of the narrow open field corridor that traces the separatrix surface of the AR flux system.

The global evolution of the magnetic and kinetic energies during the simulation are shown in Figure 3. The left panel plots the total kinetic energy $\left(E_{\mathrm{K}}\right.$, solid line) and the free magnetic energy (dashed line) within the entire computational domain. The light-gray bar indicates the time interval shown in Figure 2. We define the change in magnetic energy from the initial, potential state as $\Delta E_{\mathrm{M}}=E_{\mathrm{M}}(t)-E_{\mathrm{M}}(0)$ with $E_{\mathrm{M}}(0)=2.597 \times 10^{33} \mathrm{erg}$. Since the normal magnetic-flux distribution is fixed throughout the simulation, $\Delta E_{\mathrm{M}}$ represents the free magnetic energy of the system above the initial potential state. The amount of free magnetic energy released via the IR process $\left(\sim 10^{31} \mathrm{erg}\right)$ is only $\sim 10 \%$ of the maximum stored value. In terms of the total free energy in the system, a relatively small amount of magnetic energy release can result in significant topological restructuring and the wave generation we will examine herein.

The right panel of Figure 3 plots the evolution of the open flux. We calculated the open flux at each simulation output time in a spherical subvolume above the AR defined as $r \in\left\{1 R_{\odot}, 3 R_{\odot}\right\}, \theta \in\{0.540,1.915\} \mathrm{rad}$, and $\phi \in$ $\{-0.785,+0.785\}$ rad. First, we integrated a large number of magnetic field lines ( $\gtrsim 65000)$ from both the lower and upper boundaries and tested whether each 

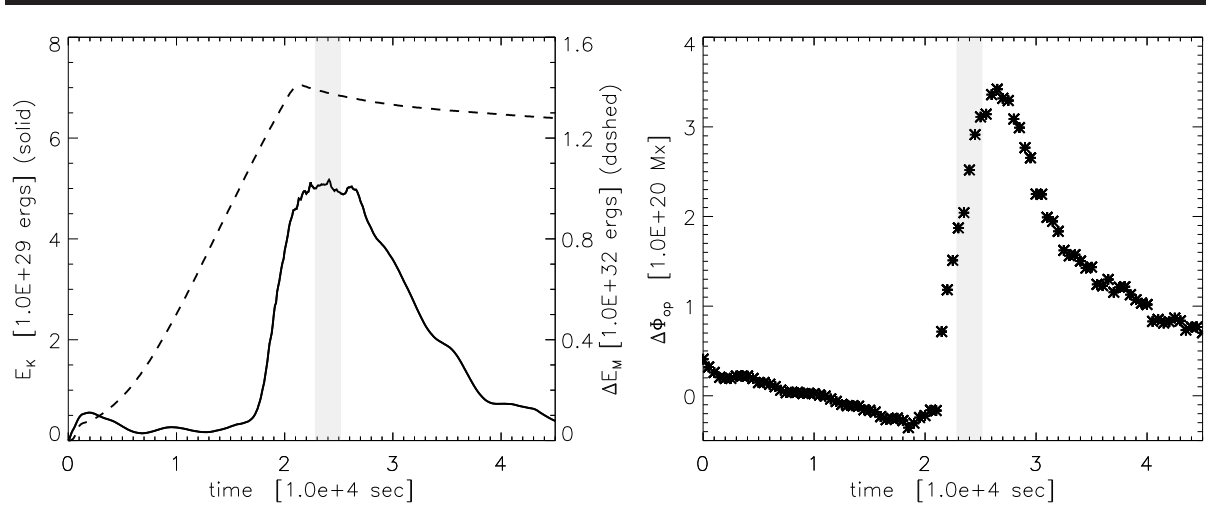

Figure 3. Left panel: kinetic energy $E_{\mathrm{K}}$ (solid) and the free magnetic energy $\Delta E_{\mathrm{M}}$ (dashed) evolution during the simulation (adapted from Edmondson et al., 2009). Right panel: change in open flux $\Delta \Phi_{\mathrm{op}}=\Phi_{\mathrm{op}}-\left\langle\Phi_{0}\right\rangle$ in a spherical subdomain. The light-gray bar indicates the time period of interchange reconnection that opens previously closed flux shown in Figure 2.

fieldline is open, i.e., has one end terminate at the lower boundary and the other at the upper boundary. Next, we constructed a pixel mask of the location of open field footpoints (bottom row of Figure 2), calculated the radial flux associated with each open field pixel, and then summed the total to obtain an estimate of the open flux:

$$
\Phi_{\mathrm{op}}=\sum B_{r}\left(R_{\odot}, \theta_{j}, \phi_{i}\right) \Delta A_{i j}
$$

where $\Delta A_{i j}=R_{\odot}^{2} \sin \theta_{j} \Delta \theta \Delta \phi, \Delta \theta=0.0052 \mathrm{rad}$, and $\Delta \phi=0.0061 \mathrm{rad}$. We define the change in open flux as $\Delta \Phi_{\mathrm{op}}=\Phi_{\mathrm{op}}(t)-\left\langle\Phi_{0}\right\rangle$, where $\left\langle\Phi_{0}\right\rangle=4.43 \times$ $10^{21} \mathrm{Mx}$ is the mean background open flux in our spherical sub-volume, taken over from $t=0$ through $t=20000 \mathrm{~s}$.

Interchange reconnection conserves the global flux topologies of the various structures, so the increase seen in the subvolume is offset by open flux closing down elsewhere in the computational domain, and we did not count flux associated with field lines that pass through either of the $r-\theta$ or $r-\phi$ planar boundaries of our subvolume. The linear decrease in open flux for $t \leq 20000 \mathrm{~s}$ is due to the gradual reconnection of the overlying field in response to the expansion of the AR flux system which drives a deformation of the null-point into a reconnecting current sheet.

Following reconnection onset, the unsheared portion of the AR flux overlying the southern section of the polarity inversion line is gradually transferred to the northern half. In addition, the reconnection process transfers the (globally closed) streamer-belt flux to the southern side of the AR flux system, shifting the AR flux system ever closer to the global streamer belt - coronal hole boundary. This closed-closed reconnection has the effect of both shifting the geometric identity (e.g., particular set of field lines at any given time) of the AR separatrix boundary and the outer spine line closer to the open-closed field interface (e.g., Edmondson et al., 2009, 2010b). Given our spherical subvolume and the method used to classify open field lines, this evolution appears as a gradual decrease of open flux. 
The evolution of the open flux has a qualitative resemblance to the kinetic energy curve. The kinetic energy increases sharply at $t \sim 18000 \mathrm{~s}$ (indicating the onset of relatively fast reconnection) whereas the change in open flux lags slightly, starting its rapid rise at $t \sim 21000 \mathrm{~s}$. We open approximately $3 \times 10^{20} \mathrm{Mx}$ of AR flux over the $10000 \mathrm{~s}$ it takes for the system to relax enough to start to close this flux back down. The maximum peak in $E_{\mathrm{K}}$ curve is reached by $22000 \mathrm{~s}$, while $\Delta \Phi_{\text {op }}$ reaches its maximum peak by $26000 \mathrm{~s}$.

\section{Material, Energy, and Helicity Flux Estimates}

We quantified the material, energy, and helicity fluxes resulting from our IR generated outflow by examining the fluxes through a radial surface of the spherical subvolume defined in $\S 2.2$ at $r=2 R_{\odot}$. The total rate of change in mass, kinetic energy, enthalpy, magnetic energy, and magnetic helicity passing through this surface is then given by integrating their respective flux densities,

$$
\begin{aligned}
\frac{d M}{d t} & =\sum \rho v_{r} \Delta A_{i j}, \\
\frac{d E_{\mathrm{K}}}{d t} & =\sum\left(\frac{1}{2} \rho v^{2}\right) v_{r} \Delta A_{i j}, \\
\frac{d E_{\mathrm{E}}}{d t} & =\sum\left(5 n k_{B} T\right) v_{r} \Delta A_{i j}, \\
\frac{d E_{\mathrm{M}}}{d t} & =\sum S_{r} \Delta A_{i j},
\end{aligned}
$$

where the sum is over $\left(\theta_{i}, \phi_{j}\right)$ elements, $\Delta A_{i j}$ is defined in Equation (1) at $r=$ $2 R_{\odot}$, the number density is $n=\rho / m_{p}$, and $k_{B}$ is the Boltzmann constant. The radial component of the Poynting flux $S_{r}$ is calculated from $\mathbf{S}=(4 \pi)^{-1} c \mathbf{E} \times \mathbf{B}$ with the electric field $c \mathbf{E}=-\mathbf{v} \times \mathbf{B}$ given by the advection velocity, yielding,

$$
S_{r}=\frac{1}{4 \pi}\left[\left(B_{\theta}^{2}+B_{\phi}^{2}\right) v_{r}-\left(B_{\theta} v_{\theta}+B_{\phi} v_{\phi}\right) B_{r}\right] .
$$

We note that the first term corresponds to the radial transport of magnetic energy associated with the tangential field components $\left(B_{\theta}, B_{\phi}\right)$ carried through the surface by $v_{r}$, and the second term describes the energy flux associated with radial fields experiencing lateral transport (see Abbett and Fisher, 2012 for discussion). In general, attributing components of the magnetic energy flux to well-defined wave-modes (see Section 4) in systems with complex magnetic configurations is not straightforward. However, as we describe below, the largest, positive $S_{r}$ enhancement corresponds spatially and temporally with the positive outflow of the other material and energy quantities.

We also calculated the flux of relative helicity through our surface (e.g., Berger and Field, 1984; Berger and Ruzmaikin, 2000),

$$
\frac{d H}{d t}=2 \oint_{S} d S\left(\mathbf{A}_{p} \cdot \mathbf{v}\right) B_{r}-\left(\mathbf{A}_{p} \cdot \mathbf{B}\right) v_{r} .
$$


where $\mathbf{A}_{p}$ is the vector potential of the reference field (e.g., potential field for given boundary conditions). The vector potential $\mathbf{A}_{p}$ was calculated using the flux function, $\mathbf{A}_{p}=\hat{\mathbf{r}} \times \nabla \psi$ from the $B_{r}$ distribution at $r=2 R_{\odot}$. In spherical coordinates, the flux function is given by

$$
\psi=-\frac{1}{4 \pi} \oint_{S^{\prime}} d S^{\prime} B_{r}\left(\theta^{\prime}, \phi^{\prime}\right) \ln \left[\frac{1-\cos \xi}{2}\right],
$$

with $\cos \xi$ in the natural logarithm term being the spherical (angular) distance between $(\theta, \phi)$ and $\left(\theta^{\prime}, \phi^{\prime}\right)$ given by Berger and Ruzmaikin (2000) as

$$
\cos \xi=\cos \theta \cos \theta^{\prime}-\sin \theta \sin \theta^{\prime} \cos \left(\phi-\phi^{\prime}\right) .
$$

Here, $d S$ and $d S^{\prime}$ correspond to the same surface area differentials as in Equations (2)-(5). The physical interpretation of this double integral is the total magnetic-field rotation at each surface point, due to both the rotation of tangential flows and twisted flux transported by radial flows. We note that this calculation is the spherical version of the Cartesian formalism used to quantify the helicity expulsion in the Pariat, Antiochos, and DeVore $(2009,2010)$ coronal jet simulations.

Figure 4 plots in rows (a) the mass flux, (b) kinetic energy flux, and (c) the enthalpy flux densities on the $2 R_{\odot}$ radial surface of our spherical subdomain at the simulation time $t=25000 \mathrm{~s}$ in the left panels and the temporal evolution of the surface integral quantities $d M / d t, d E_{\mathrm{K}} / d t$, and $d E_{\mathrm{E}} / d t$ (given by Equations (2)(4)) that represent the time rate of change of each of these quantities passing through the radial surface. We have plotted both the positive (negative) material and energy quantities separately as the thick solid (dotted) lines, respectively. The net totals are shown as the dashed lines. The linear scaling of the integrated energy plots masks the small but non-zero kinetic energy flux $d E_{k} / d t$ asocialted with the non-zero mass fluxes $d M / d t$ before the onset of the main phase of IR, but the magnitude is only $\sim 10^{21} \mathrm{ergs} \mathrm{s}^{-1}$ for $t<20000 \mathrm{~s}$.

Figure 5 plots in row (a) the magnetic energy flux density and in row (b) the relative helicity flux density and their surface integral totals (Equations (5) and (7), respectively) in the same format as Figure 4. For the time rate of change in magnetic helicity we flipped the $y$-axis values and show the negative/left-hand helicity flux as the solid line to facilitate the visual association with the positive material and energy IR-jet outflow quantities.

The strong positive flux density enhancements in mass density, kinetic energy, enthalpy, and magnetic energy flux densities directly correspond to the interchange reconnection jet outflow. Likewise, the strong negative helicity flux density is aligned co-spatially with the other outflow quantities, and represents the positive radial transport of left-hand twist on newly opened magnetic field lines (e.g., the un-twisting of the field stressed by counter-clockwise rotational driving flows). Once the IR starts in earnest at $\sim 20000 \mathrm{~s}$ (visible in Figure 3), the outflow signatures appear at $(-0.2,+0.1) \mathrm{rad}$ in latitude, longitude and propagate slightly eastward and towards the northern pole. The outflow flux spatial distributions are complicated after $\gtrsim 30000 \mathrm{~s}$ because of the signal reflections off of the outer boundary. 



Figure 4. Flux densities through the $r=2 R_{\odot}$ Gaussian surface at $t=25000 \mathrm{~s}$ : (a) mass flux, (b) kinetic energy flux, (c) enthalpy flux. The right column plots the surface integrals corresponding to the time rate of change in mass, kinetic energy, and enthalpy through the radial surface (Equations (2)-(4)) where the positive fluxes are shown as thick solid lines, negative fluxes as dotted lines, and the net flux as dashed lines.

We note that there are significant downflows of both mass and energy present in addition to the expected IR generated outflows. These are largely due to gravity acting on displaced material. A back-of-the-envelope calculation of the free fall speeds confirm these inflows as simply material flowing down recently "straightened out" field lines that have either newly opened or recently reconnected. In the absence of a background solar-wind outflow, only the material accelerated by the reconnection jet and carried by the associated waves continue to propagate radially outwards. 

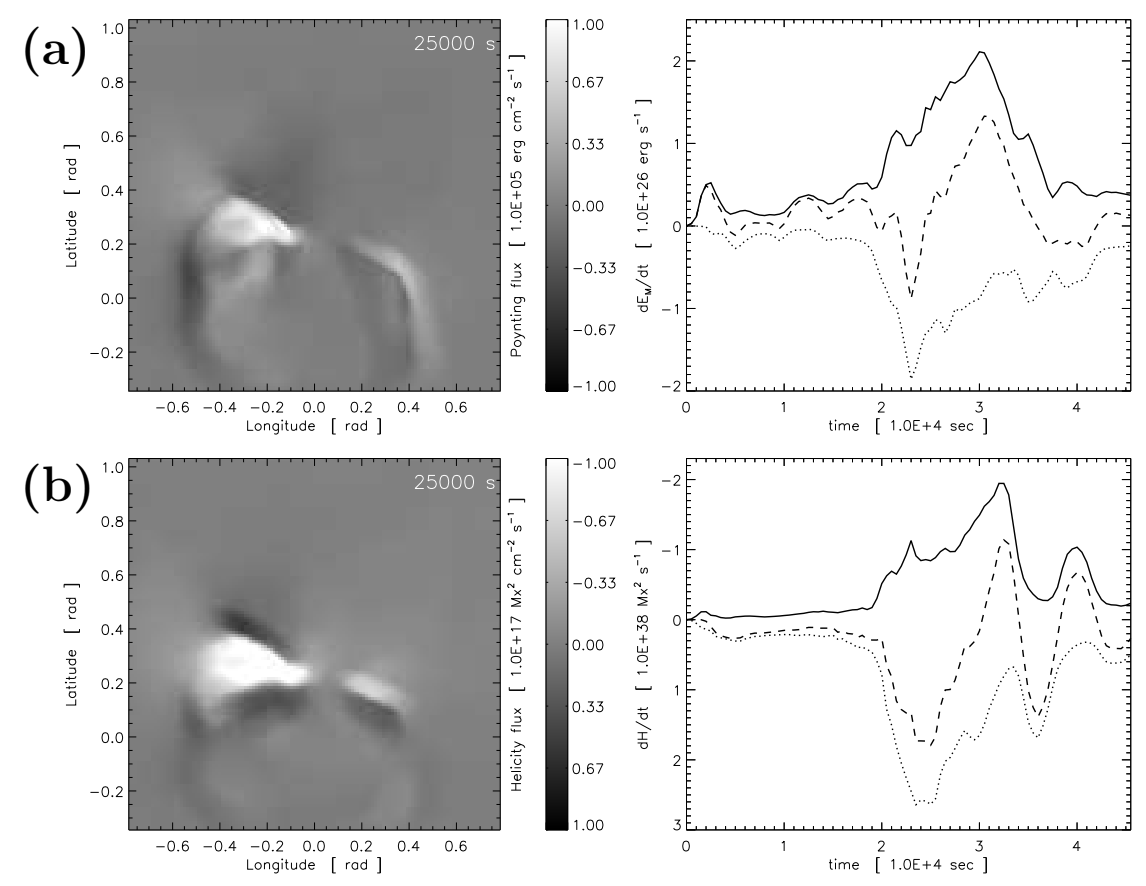

Figure 5. Flux densities for the (a) Poynting flux and (b) relative helicity flux in the same format as Figure 4. The right panels plot the temporal evolution of the surface integral of each quantity (Equation (5) and (7)). For the total helicity rate the $y$-axis values are reversed to align the high flux of negative (left-handed) helicity with the reconnection material and energy outflow, thus the negative (positive) flux is shown as thick solid (dotted) lines, respectively.

The overall spatial distribution and evolution of the outwardly propagating mass and energy fluxes are qualitatively similar to the 3D coronal jet results presented by Pariat, Antiochos, and DeVore (2009), although as we discussed previously (Edmondson et al., 2009), this simulation is much less "explosive" because the injected stresses are closer to the AR separatrix and therefore allows the release of accumulated magnetic energy through current sheet generation and reconnection much earlier; only a small amount of the magnetic free energy is released. The vast majority of the accumulated relative helicity also remains enclosed in the AR flux system. The relative helicity flux introduced during the uniform phase of the vortical shearing flows at the $r=R_{\odot}$ lower boundary was $d H / d t=-1.90 \times 10^{39} \mathrm{Mx}^{2} \mathrm{~s}^{-1}$. Thus, the magnitude of both the positive and negative helicity fluxes through the $2 R_{\odot}$ surface from the IR process are only on the order of $5-10 \%$ of that associated with the energizing footpoint motions.

It is also interesting to compare the ratio of maximum values of the integrated magnetic with kinetic energy fluxes carried by the jet outflow in the two simulations: the Pariat, Antiochos, and DeVore (2009) results yield a more kinetic jet $d E_{\mathrm{M}}: d E_{\mathrm{K}} \sim 4: 1$, whereas our results yield $d E_{\mathrm{M}}: d E_{\mathrm{K}} \sim 10: 1$. In both cases the torsional Alfvén waves provided the bulk of the magnetic energy transport away from the reconnection region, but the Pariat, Antiochos, and 
DeVore (2009) jet opens more of the closed flux and is able to transfer $90 \%$ of the total introduced helicity from the closed to open field lines, whereas our IR "relaxation" transfers roughly 5\%. It is also important to recall that for planar Alfvén waves the magnetic and kinetic energy densities are in equipartition, so our results indicate the significance of the additional magnetic energy transport associated with opening up previously closed magnetic flux.

\section{Reconnection-Driven Alfvén Waves}

\subsection{Low-Frequency Waves from IR Topological Restructuring}

The basic topology and evolution of the generation of large-scale, nonlinear torsional Alfvén waves associated with interchange reconnection opening up twisted closed flux are apparent from Figure 2 as well as in the online animations of Edmondson et al. (2009). To characterize the wave properties of the outwardly propagating disturbances, we define the magntiude of the magnetic field fluctuations as

$$
\frac{\delta B}{\langle B\rangle}=\frac{\left|\mathbf{B}(t)-\langle\mathbf{B}\rangle_{t}\right|}{\langle B\rangle_{t}} .
$$

Here, our background "average" field $\langle\mathbf{B}\rangle_{t}$ is computed at a fixed position from the running temporal average of the magnetic field centered on $t_{i}$ with an averaging window width of $2000 \mathrm{~s}$. For our simulation output cadence this represents five data files $(-2 \Delta t$ through $+2 \Delta t)$ and the window width is similar to the global characteristic Alfvén time $\left\langle\tau_{A}\right\rangle=2 R_{\odot} /\left\langle V_{A}\right\rangle$ calculated in Edmondson et al. (2009). In the limit of small fluctuations, Equation (10) reduces to the standard formulation used in linearized perturbation analysis.

Figure 6 plots the $r-\theta$ plane of our normalized field fluctuation quantity $\delta B /\langle B\rangle$ for three longitudinal values indicated as dashed green lines in panel (a). Panels (b), (c), and (d) show the planes for $\phi$ values of $\{-32,-22,0\}$ degrees, respectively. These planar cuts sample the open field corridor that initially opens on the eastern side of the AR separatrix dome. The animation of this figure (included as an electronic supplement to the online version of this article) shows the formation and propagation of the torsional wave, illustrated most clearly in panel (c). The twisted/helical structure of the propagating wave packet, i.e. strong regions of oppositely directed $\delta B_{\phi}$, creates the dual-peak signature in the fluctuation magnitude plots. One can compare the $\delta B /\langle B\rangle$ visualization here with the material and energy fluxes in Section 3 (Figure 4), which corresponds to the midpoint of the $x$-axis range. The flux evolution of $E_{\mathrm{K}}, E_{\mathrm{M}}$, and $H_{r}$ can be seen as the intersection of the torsional Alfvén wave passing through the $r=2 R_{\odot}$ radial surface.

After visualizing the coherent wave packet generation and propagation in our 3D MHD simulation data, we now examine the geometric extent and spectral properties of the wave train associated with our idealized "discrete" IR event. We expect some aspects of the spatial size of the $\delta B /\langle B\rangle$ signal to be related to the geometry of our system - specifically the size of the energized AR separatrix, 



Figure 6. Panel (a) shows $B_{r}$ in the same format as Figure 2 with the $\phi$ locations of the three $r-\theta$ planes indicated with green dashed lines for $t=25000 \mathrm{~s}$. Panels (b), (c), and (d) plot the normalized fluctuations $\delta B /\langle B\rangle$ that show the spatial extent and propagation of the large-scale nonlinear torsional Alfvén wave through the domain. An animation of this figure is available as an electronic attachment to the online version of this article.

as well as the global magnetic-field structure. As seen in Figure 6, the central region of the propagating twist wave (panel c) is between latitude values of $[-0.2,+0.2] \mathrm{rad}$. Here, the double peaks of the $\delta B /\langle B\rangle$ magnitude structure each have a characteristic width of approximately $\lambda_{r} \sim 0.2-0.25 R_{\odot}$ corresponding to a full wavelength in $\delta B_{\phi}$ of $\lambda_{r} \sim 0.4-0.5 R_{\odot}$.

Figure 7 plots the normalized fluctuation power as a function of the radial spatial frequency (simply inverse wavelength) $\nu=\lambda_{r}^{-1}$ at $t=25000 \mathrm{~s}$ for each of the three $r-\theta$ planes in Figure 6 . Here, panels (b)-(d) plot the $\theta$-distribution of the $\delta B /\langle B\rangle$ power spectra. The most common characteristic of power spectra of all three planar cuts is that for the most part, the relatively strong power is in the low spatial frequencies (longer wavelengths), demonstrating that the wavelengths generated by this isolated IR process are relatively long. An animation of Figure 7 showing the temporal evolution of the normalized power spectra is included as an electronic attachment to the online version of the article.

Upon closer examination, the power spectra through all three planar cuts exhibits a bimodal distribution in latitude, with strong power structures extending through the lower spatial frequencies, peaking in the angular ranges 

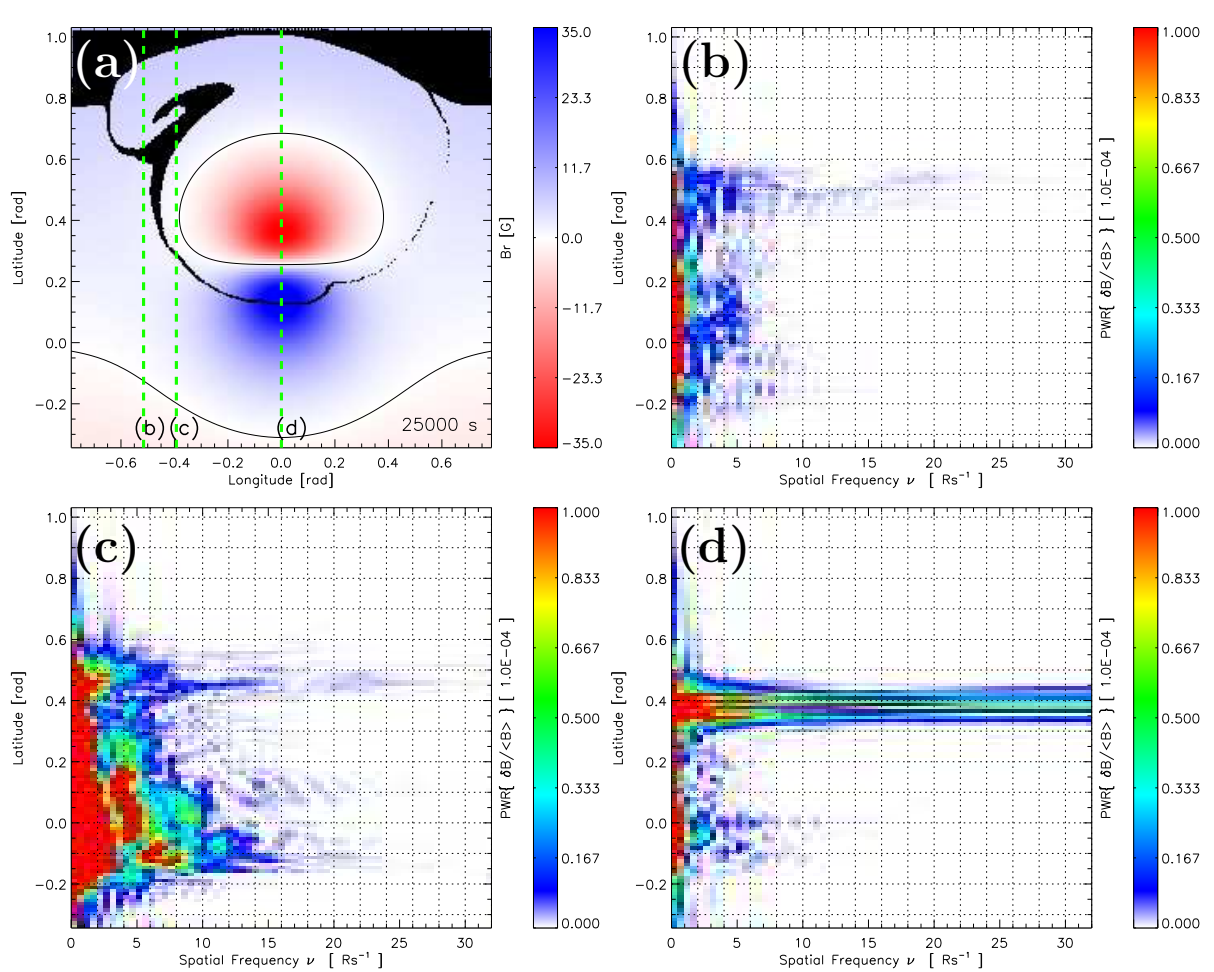

Figure 7. Panel (a) again indicates $B_{r}$ and the open flux regions. Panels (b)-(d) show the power spectra of $\delta B /\langle B\rangle$ depicted in Figure 6. The sharp, filamentary changes in the field associated with the separatrix evolving correspond to power across a broad range of $\nu$ including the highest spatial frequencies, whereas the propagating, large-scale torsional wave are clearly visible as low spatial frequency enhancements. An animation of this figure is available as an electronic attachment to the online version of this article.

$[-0.2,+0.2] \mathrm{rad}$, and $[+0.4,+0.5] \mathrm{rad}$, respectively. Physically, the bimodal distribution reflects the planar cuts sampling the different field topologies: open coronal hole, globally closed streamer belt, and locally closed AR. The relatively narrow, higher latitude peaked distribution reflects the reconnection-driven wave patterns directly related to the reconnection site, and opening of the coronal hole channel and the generation of the open field "thumb" structure on the northwest side of the AR (clearly seen in panel (a)). We note that there is a systematic shift of approximately 0.1 radians to lower latitudes with respect to the open field footpoint pattern of panel (a), due to the global magnetic geometry of the streamer belt - coronal hole pattern acting as an equatorward wave-guide for the system dynamics above the surface. On the other hand, the relatively broad, lower latitude peaked distribution reflects the reconnection-driven wave patterns of the global restructuring of the streamer belt, as the AR shifts into the coronal hole.

The strongest power exhibited in all three planar cuts is at the lowest spatial frequency $\nu \lesssim 1$, suggesting that the dominant wavelength is directly related to to the size of the separatrix dome; estimated by the angular spread between foot- 
points plotted in panel (a), the widest part at latitude $\sim 0.4$, the angular width stretches from approximately -0.5 through +0.5 in longitude, corresponding to a characteristic size of $\sim 1 R_{\odot}$. We note that this is only an estimate and does not account for height or writhe, which serve to increase the length of newly reconnected field, decreasing the corresponding spatial frequency.

The strong power in the lower latitude range, $[-0.2,+0.2] \mathrm{rad}$ has a broad angular extent and remains in the lower spatial frequency range very simply due to the global size of the streamer belt. The upper boundary of this distribution, latitude of $+0.2 \mathrm{rad}$ is approximately the lower extent of the AR structure. Thus, the broad power reflects the restructuring of the streamer belt as the AR shifts into the coronal hole, generating strong wavelength patterns in the range of order the size of the AR down to the length of the reconnecting current sheet; all three panels offer evidence that this lower power distribution broadly extends to the same spatial frequency level at the upper power distribution. That the strongest waves are shown in panel (c) reflects the fact that the driving flow is counter-clockwise, causing the IR process to move in a western direction (see Edmondson et al., 2009 for details), generating the bulk of the streamer-belt restructuring wave activity on the western limb of the AR structure.

The large-scale torsional Alfvén wave signal, most visible in panel (c), associated with the coronal hole boundary jump across the AR, generates the wave power feature at approximately $\nu \lesssim 7 / R_{\odot}$ at latitude $-0.1 \mathrm{rad}$, as well as the clear extended peak in latitude around approximately $\nu \sim 4 / R_{\odot}$ between latitudes $(-0.05,+0.2) \mathrm{rad}$. We note that the range of $\delta B /\langle B\rangle$ spatial scales associated with our interchange reconnection wave activity $\left(7 / R_{\odot} \lesssim \nu \lesssim 2 / R_{\odot}\right.$; $100 \mathrm{Mm} \lesssim \lambda_{r} \lesssim 350 \mathrm{Mm}$ ) fall within the range of the nonturbulent periodic density structures observed in the STEREO/SECCHI data remotely and in the solar wind in-situ (Viall et al., 2010), as well as comfortably within the lowfrequency range of the interplanetary Alfvén wave spectra (e.g., Belcher and Davis, 1971).

\subsection{High-Frequency Waves from the IR Current Sheet}

In all three Figure 7 planar cuts, there is also strong power in the upper latitude range, $[+0.4,+0.5] \mathrm{rad}$, extending to higher spatial frequencies, relative to the lower latitude distribution. This effect reflects a direct connection to the reconnection site that generates waves at higher spatial frequencies. From the corresponding panels in Figure 6, the spatial scale of the reconnection site (located in the range $r \sim 1.5-1.7 R_{\odot}$ and co-latitude $\sim 0.4-0.5 \mathrm{rad}$ depending on the panel) is estimated to be $L \sim 0.2-0.3 R_{\odot}$ corresponding to a spatial frequency of $\nu \sim 7.5 / R_{\odot}$; the effective upper spatial frequency limit of the relatively broad upper latitude strong power distribution of panels (b) and (c). In each panel, there is evidence of much narrower power distribution extending to even higher spatial frequencies. This effect is a remanent of even smaller scale structures within the reconnecting current sheet itself, down to the grid scale of the simulation. In fact, such high spatial frequency effects are abundant in panel (d), since this plane directly cuts the reconnection current sheet, and therefore samples the smallest scale structures of the simulation. 
We note that because highest frequency waves originate from reconnection sites, the computational grid scale and the magnetic resistivity employed place an artificial upper limit on the wave frequencies that we are able to resolve in the simulation. The tearing and breakup of currents sheets generate a whole distribution of magnetic islands, and the associated high-frequency waves generated will have characteristic wavelengths on the order of the size of the islands (Drake et al., 2006; Isobe, Proctor, and Weiss, 2008; Ji and Daughton, 2011; Bárta et al., 2011; Shen et al., 2013). Much higher resolution simulations will be needed to resolve the higher frequency wave modes. Recent studies of current sheet formation and reconnection with adaptive grid refinement (Edmondson et al., 2010a; Karpen, Antiochos, and DeVore, 2012) are beginning to resolve the current sheet substructure and island formation in global MHD models while employing a "numerical resistivity" model. However, to characterize the highest frequency wave components it will be important to investigate the effects of the magnetic resistivity model on the evolution of the current sheet evolution and reconnection. We have focused on the low-frequency torsional Alfvénic wave activity because our large-scale system evolution should be relatively independent of the specific details of the magnetic reconnection. All we require here is that some moderate amount of previously closed flux that has accumulated a significant twist/shear component is allowed to become open.

\section{Summary and Discussion}

We have presented a detailed analysis of the Edmondson et al. (2009) MHD simulation of closed-to-open interchange reconnection in an idealized AR flux system at the edge of the helmet streamer-belt. Despite the limitations of the simulation, i.e., the extremely large AR source and laminar vortical energization flows, our time-integrated IR-driven Poynting flux carries only $\sim 1 \%$ of the accumulated free magnetic energy introduced into the system yet generates significant coronal dynamics; notably the generation of a large-scale, torsional Alfvén wave that transmits the shear and twist components of the energized, previously closed AR flux into the open field region. We visualized this large-scale wave activity as a succession of field-line plots (Figure 2), as well as the formation and propagation of the $\delta B /\langle B\rangle$ structure (Figure 6 and its online animation). One of our primary goals was to emphasize the intrinsic and fundamental connection between reconnection and Alfvén -wave generation.

Large-scale torsional Alfvén waves have been identified in previous simulation work on coronal jets (Shibata and Uchida, 1986; Pariat, Antiochos, and DeVore, 2009) and flux emergence (Török et al., 2009), and appear to explain recent observations that show evidence of helical structure or apparent twisting/un-twisting motions (Patsourakos et al., 2008; Liu et al., 2009; Kamio et al., 2010; Shen et al., 2011), and may be a significant component of the ubiquitous wave activity observed (e.g., Cirtain et al., 2007). Observations have shown that impulsive, jet-like processes are operating over a wide range of spatial scales in both open- and closed-field regions and in different layers of the atmosphere, including photospheric and chromospheric jets and spicules (De 
Pontieu et al., 2007; Sterling, Harra, and Moore, 2010; Liu et al., 2011a), H $\alpha$ surges (Kurokawa and Kawai, 1993; Canfield et al., 1996), low coronal jets in EUV (Chae et al., 1999; Jiang et al., 2007; Patsourakos et al., 2008) and X-rays (Shibata et al., 1997; Alexander and Fletcher, 1999; Cirtain et al., 2007). Liu et al. (2011a, 2011b) have measured both the stationary component where the entire jet appears to move back and forth, which is indicative of transverse footpoint motion, as well as the impulsive, outwardly propagating wave transients driven by reconnection - and similar features have been reproduced in numerical simulations of (Moreno-Insertis, Galsgaard, and Ugarte-Urra, 2008; Murray, van Driel-Gesztelyi, and Baker, 2009). At all spatial scales, magnetic reconnection processes drive Alfvén waves into the corona and in unipolar open field regions, ultimately into the solar wind.

Recently, Cranmer and van Ballegooijen (2010) constructed a kinematic model that included both flux emergence and quasi-static evolution to quantify the energy fluxes generated by IR process and concluded that IR has trouble producing values within the range of a few $\times 10^{5}-10^{6} \mathrm{erg} \mathrm{cm}^{-2} \mathrm{~s}^{-1}$ needed to accelerate the solar wind. As shown in Figure 4, our calculation of the radial Poynting flux through a Gaussian surface above the IR site readily produces $10^{5} \mathrm{erg} \mathrm{cm}^{-2} \mathrm{~s}^{-1}$ for the duration of the dynamic flux opening ( $\sim 2.5$ hours). The radial Poynting flux carried by our singular interchange reconnection event "jet" subtends an angular area of $\sim 0.20 \%$ of the $4 \pi$ spherical surface. However, our simulation probably represents the largest conceivable scale IR processes operate on before being considered a slow solar wind "streamer blob" and the fluctuations of these wavelengths are likely to escape directly into the solar wind without contributing much to the heating process.

To argue that IR could supply all of the necessary power to heat the corona and accelerate the solar wind, one would have to (1) determine how the IR-driven Alfvén wave properties scale with bipolar flux system properties (field strength, flux content, geometric size) and energization mechanism (rotational and translational motions, footpoint shuffling associated with granular and super-granular diffusion), and (2) estimate the distribution of bipolar flux system source sizes from high-resolution coronal magnetic-field extrapolations. If IR were a continuous stochastic, intermittent process and the IR-driven wave power and characteristic wavelengths reflected their source region structure, then one could estimate this contribution to the low-frequency portion of the observed interplanetary Alfvén wave spectra. Since the topological structure of nested flux systems with a complex network of separatrices and quasi-separatricies (favorable sites of IR) is exactly what high-resolution PFSS extrapolations and the resulting Q-maps show (Linker et al., 2011; Titov et al., 2011) and the basis of the Antiochos et al. (2011) S-Web model - we are looking forward to future progress in this arena.

Acknowledgements BJL and YL acknowledge support from the AFOSR YIP FA955011-1-0048, NASA HTP NNX11AJ65G, and HGI NNX08AJ04G. JKE acknowledges support NASA LWS NNX10AU57G and NNX07AB99G.

\section{References}

Abbett, W.P., Fisher, G.H.: 2012, Solar Phys. 277, 3. doi:10.1007/s11207-011-9817-3. 
Alexander, D., Fletcher, L.: 1999, Solar Phys. 190, 167. doi:10.1023/A:1005213826793.

Antiochos, S.K.: 2013, Astrophys. J. 772, 72. doi:10.1088/0004-637X/772/1/72.

Antiochos, S.K., Mikić, Z., Titov, V.S., Lionello, R., Linker, J.A.: 2011, Astrophys. J. 731, 112. doi:10.1088/0004-637X/731/2/112.

Bárta, M., Büchner, J., Karlický, M., Skála, J.: 2011, Astrophys. J. 737, 24. doi:10.1088/0004-637X/737/1/24.

Belcher, J.W., Davis, L. Jr.: 1971, J. Geophys. Res. 76, 3534. doi:10.1029/JA076i016p03534.

Berger, M.A., Field, G.B.: 1984, Journal of Fluid Mechanics 147, 133. doi:10.1017/S0022112084002019.

Berger, M.A., Ruzmaikin, A.: 2000, J. Geophys. Res. 105, 10481. doi:10.1029/1999JA900392.

Bryans, P., Landi, E., Savin, D.W.: 2009, Astrophys. J. 691, 1540. doi:10.1088/0004-637X/691/2/1540.

Canfield, R.C., Reardon, K.P., Leka, K.D., Shibata, K., Yokoyama, T., Shimojo, M.: 1996, Astrophys. J. 464, 1016. doi:10.1086/177389.

Chae, J., Qiu, J., Wang, H., Goode, P.R.: 1999, Astrophys. J. 513, L75. doi:10.1086/311910.

Cirtain, J.W., Golub, L., Lundquist, L., van Ballegooijen, A., Savcheva, A., Shimojo, M., DeLuca, E., Tsuneta, S., Sakao, T., Reeves, K., Weber, M., Kano, R., Narukage, N., Shibasaki, K.: 2007, Science 318. doi:10.1126/science.1147050.

Cranmer, S.R., van Ballegooijen, A.A.: 2010, Astrophys. J. $\mathbf{7 2 0 ,} 824$. doi:10.1088/0004-637X/720/1/824.

Cranmer, S.R., van Ballegooijen, A.A., Edgar, R.J.: 2007, Astrophys. J. Suppl. 171, 520. doi:10.1086/518001.

De Pontieu, B., McIntosh, S.W., Carlsson, M., Hansteen, V.H., Tarbell, T.D., Schrijver, C.J., Title, A.M., Shine, R.A., Tsuneta, S., Katsukawa, Y., Ichimoto, K., Suematsu, Y., Shimizu, T., Nagata, S.: 2007, Science 318. doi:10.1126/science.1151747.

DeVore, C.R.: 1991, Journal of Computational Physics 92, 142. doi:10.1016/0021-9991(91)90295-V.

DeVore, C.R., Antiochos, S.K.: 2008, Astrophys. J. 680, 740. doi:10.1086/588011.

Drake, J.F., Swisdak, M., Che, H., Shay, M.A.: 2006, Nature 443, 553. doi:10.1038/nature05116.

Edmondson, J.K., Lynch, B.J., Antiochos, S.K., De Vore, C.R., Zurbuchen, T.H.: 2009, Astrophys. J. 707, 1427. doi:10.1088/0004-637X/707/2/1427.

Edmondson, J.K., Antiochos, S.K., DeVore, C.R., Zurbuchen, T.H.: 2010a, Astrophys. J. 718, 72. doi:10.1088/0004-637X/718/1/72.

Edmondson, J.K., Antiochos, S.K., DeVore, C.R., Lynch, B.J., Zurbuchen, T.H.: 2010b, Astrophys. J. 714, 517. doi:10.1088/0004-637X/714/1/517.

Fisk, L.A.: 2005, Astrophys. J. 626, 563. doi:10.1086/429957.

Fisk, L.A., Schwadron, N.A.: 2001, Astrophys. J. 560, 425. doi:10.1086/322503.

Fisk, L.A., Zurbuchen, T.H.: 2006, Journal of Geophysical Research (Space Physics) 111(A10), 9115. doi:10.1029/2005JA011575.

Fisk, L.A., Zurbuchen, T.H., Schwadron, N.A.: 1999, Astrophys. J. 521, 868. doi:10.1086/307556.

Geiss, J., Gloeckler, G., von Steiger, R.: 1995, Space Sci. Rev. 72, 49. doi:10.1007/BF00768753.

Gosling, J.T.: 1997, In: S. R. Habbal (ed.) Robotic Exploration Close to the Sun: Scientific Basis, Am. Inst. Phys. CS-385, 17. doi:10.1063/1.51743.

Hollweg, J.V.: 1986, J. Geophys. Res. 91, 4111. doi:10.1029/JA091iA04p04111.

Isobe, H., Proctor, M.R.E., Weiss, N.O.: 2008, Astrophys. J. 679, L57. doi:10.1086/589150.

Ji, H., Daughton, W.: 2011, Physics of Plasmas 18(11), 111207. doi:10.1063/1.3647505.

Jiang, Y.C., Chen, H.D., Li, K.J., Shen, Y.D., Yang, L.H.: 2007, Astron. Astrophys. 469, 331. doi:10.1051/0004-6361:20053954.

Kamio, S., Curdt, W., Teriaca, L., Inhester, B., Solanki, S.K.: 2010, Astron. Astrophys. 510, L1. doi:10.1051/0004-6361/200913269.

Karpen, J.T., Antiochos, S.K., DeVore, C.R.: 2012, Astrophys. J. $\mathbf{7 6 0 ,} 81$. doi:10.1088/0004-637X/760/1/81.

Kurokawa, H., Kawai, G.: 1993, In: Zirin, H., Ai, G., Wang, H. (eds.) IAU Colloq. 141: The Magnetic and Velocity Fields of Solar Active Regions, Astron. Soc. Pac. CS-46, 507.

Laming, J.M.: 2004, Astrophys. J. 614, 1063. doi:10.1086/423780.

Laming, J.M.: 2009, Astrophys. J. 695, 954. doi:10.1088/0004-637X/695/2/954.

Linker, J.A., Lionello, R., Mikić, Z., Titov, V.S., Antiochos, S.K.: 2011, Astrophys. J. 731, 110. doi:10.1088/0004-637X/731/2/110. 
Liu, W., Berger, T.E., Title, A.M., Tarbell, T.D.: 2009, Astrophys. J. 707, L37. doi:10.1088/0004-637X/707/1/L37.

Liu, W., Berger, T.E., Title, A.M., Tarbell, T.D., Low, B.C.: 2011a, Astrophys. J. 728, 103. doi:10.1088/0004-637X/728/2/103.

Liu, W., Title, A.M., Zhao, J., Ofman, L., Schrijver, C.J., Aschwanden, M.J., De Pontieu, B., Tarbell, T.D.: 2011b, Astrophys. J. 736, L13. doi:10.1088/2041-8205/736/1/L13.

MacNeice, P., Olson, K.M., Mobarry, C., de Fainchtein, R., Packer, C.: 2000, Computer Physics Communications 126, 330. doi:10.1016/S0010-4655(99)00501-9.

Matthaeus, W.H., Zank, G.P., Oughton, S., Mullan, D.J., Dmitruk, P.: 1999, Astrophys. J. 523, L93. doi:10.1086/312259.

Moreno-Insertis, F., Galsgaard, K., Ugarte-Urra, I.: 2008, Astrophys. J. 673, L211. doi: $10.1086 / 527560$.

Murray, M.J., van Driel-Gesztelyi, L., Baker, D.: 2009, Astron. Astrophys. 494, 329. doi:10.1051/0004-6361:200810406.

Ofman, L.: 2004, Journal of Geophysical Research (Space Physics) 109, 7102. doi:10.1029/2003JA010221.

Ofman, L.: 2010, Journal of Geophysical Research (Space Physics) 115, 4108. doi:10.1029/2009JA015094.

Pariat, E., Antiochos, S.K., DeVore, C.R.: 2009, Astrophys. J. 691, 61. doi:10.1088/0004-637X/691/1/61.

Pariat, E., Antiochos, S.K., DeVore, C.R.: 2010, Astrophys. J. 714, 1762. doi:10.1088/0004-637X/714/2/1762

Patsourakos, S., Pariat, E., Vourlidas, A., Antiochos, S.K., Wuelser, J.P.: 2008, Astrophys. J. 680, L73. doi:10.1086/589769.

Shen, C., Lin, J., Murphy, N.A., Raymond, J.C.: 2013, Physics of Plasmas 20(7), 072114. doi:10.1063/1.4816711.

Shen, Y., Liu, Y., Su, J., Ibrahim, A.: 2011, Astrophys. J. $735, \quad$ L43. doi:10.1088/2041-8205/735/2/L43.

Shibata, K., Uchida, Y.: 1986, Solar Phys. 103, 299. doi:10.1007/BF00147831.

Shibata, K., Shimojo, M., Yokoyama, T., Ohyama, M.: 1997, In: Bentley, R.D., Mariska, J.T. (eds.) Magnetic Reconnection in the Solar Atmosphere, Astron. Soc. Pac. CS-111, 29.

Smith, C.W.: 1999, Washington DC American Geophysical Union Geophysical Monograph Series 111, 239

Sterling, A.C., Harra, L.K., Moore, R.L.: 2010, Astrophys. J. $\mathbf{7 2 2 ,} 1644$. doi:10.1088/0004-637X/722/2/1644.

Titov, V.S., Mikić, Z., Linker, J.A., Lionello, R., Antiochos, S.K.: 2011, Astrophys. J. 731, 111. doi:10.1088/0004-637X/731/2/111.

Török, T., Aulanier, G., Schmieder, B., Reeves, K.K., Golub, L.: 2009, Astrophys. J. 704, 485. doi:10.1088/0004-637X/704/1/485.

Verdini, A., Velli, M., Matthaeus, W.H., Oughton, S., Dmitruk, P.: 2010, Astrophys. J. 708, L116. doi:10.1088/2041-8205/708/2/L116.

Viall, N.M., Spence, H.E., Vourlidas, A., Howard, R.: 2010, Solar Phys. 267, 175. doi:10.1007/s11207-010-9633-1.

Zurbuchen, T.H.: 2007, Annu. Rev. Astro. Astrophys. 45, 297. doi:10.1146/annurev.astro.45.010807.154030. 